\title{
Effects of maternal immune activation on gene expression patterns in the fetal brain
}

\author{
KA Garbett ${ }^{1,5}$, EY Hsiao ${ }^{2,5}$, S Kálmán ${ }^{1,3}$, PH Patterson ${ }^{2}$ and K Mirnics ${ }^{1,4}$
}

We are exploring the mechanisms underlying how maternal infection increases the risk for schizophrenia and autism in the offspring. Several mouse models of maternal immune activation (MIA) were used to examine the immediate effects of MIA induced by influenza virus, poly(I:C) and interleukin IL- 6 on the fetal brain transcriptome. Our results indicate that all three MIA treatments lead to strong and common gene expression changes in the embryonic brain. Most notably, there is an acute and transient upregulation of the $\alpha, \beta$ and $\gamma$ crystallin gene family. Furthermore, levels of crystallin gene expression are correlated with the severity of MIA as assessed by placental weight. The overall gene expression changes suggest that the response to MIA is a neuroprotective attempt by the developing brain to counteract environmental stress, but at a cost of disrupting typical neuronal differentiation and axonal growth. We propose that this cascade of events might parallel the mechanisms by which environmental insults contribute to the risk of neurodevelopmental disorders such as schizophrenia and autism.

Translational Psychiatry (2012) 2, e98; doi:10.1038/tp.2012.24; published online 3 April 2012

\section{Introduction}

Maternal infection is a risk factor for schizophrenia and autism. In the case of schizophrenia, a wide variety of infections during pregnancy (viral, bacterial, parasitic) are associated with increased risk for this disorder in the offspring. Summing these risks, Brown and Derkits ${ }^{1}$ estimate that $>30 \%$ of schizophrenia cases would be prevented if infection could be averted in pregnant women. The fact that such a diverse set of pathogens is associated with risk suggests that it is the mother's response to the infection that is critical for altering fetal brain development. In fact, the maternal response during gestation (elevated cytokines and anti-pathogen antibodies) is associated with the increase in schizophrenic outcome in the offspring. Similarly for autism, a study of $>10000$ cases in the Danish Medical Registry revealed an association between viral or bacterial infection in the mother and increased risk for the offspring. ${ }^{2}$ Also similar to schizophrenia are findings of an increased risk for autism in the offspring if particular cytokines or chemokines are elevated in maternal serum or amniotic fluid. ${ }^{3,4}$ The observation of a significantly higher concordance in dizygotic twins than in nontwin siblings also suggests the importance of the maternalfetal environment in autism. ${ }^{5,6}$

To mimic the environmental risk for development of schizophrenia and autism, several animal models for maternal immune activation (MIA) have been used successfully. ${ }^{7}$ First, intranasal instillation of human influenza virus in pregnant mice or non-human primates induces a moderate but sublethal infection, and the offspring display a series of histological and molecular abnormalities in the hippocampus and cortex. ${ }^{8,9}$
Young and adult mouse MIA offspring ${ }^{10}$ also exhibit a cerebellar neuropathology that is commonly found in autism. ${ }^{11,12}$ Furthermore, adult mice born to infected mothers display behavioral abnormalities that are relevant to both schizophrenia and autism, including deficits in social interaction, prepulse inhibition, open field and novel object exploration, ${ }^{10}$ as well as heightened responsivity to a hallucinogen. ${ }^{13}$ Second, MIA can also be induced in the absence of pathogens by injecting pregnant rats, mice or monkeys with the synthetic dsRNA, poly(I:C) to mimic a viral infection or with lipopolysaccharide to mimic a bacterial infection. Overall, the behavioral results from the maternal infection, poly $(\mathrm{l}: \mathrm{C})$ and lipopolysaccharide models is consistent, with many results being reproduced in both rats and mice. ${ }^{7,14,15}$ Molecular and cellular studies of adult offspring of poly(I:C)-treated rodents reveal abnormalities that are clearly relevant to schizophrenia, such as increased levels of $\mathrm{GABA}_{\mathrm{A}}$ receptor $\alpha 2$ immunoreactivity, dopamine hyperfunction, delay in hippocampal myelination, reduced NMDA receptor expression in the hippocampus, reduced numbers of reelin- and parvalbuminpositive cells in the prefrontal cortex, reduced dopamine D1 and D2 receptors in the prefrontal cortex and enhanced tyrosine hydroxlyase in striatal structures. ${ }^{16}$ Third, MIA can be induced more directly by a single injection of the cytokine interleukin IL-6 in pregnant mice. ${ }^{17}$ This approach is based on findings that this injection yields offspring displaying many of the same behavioral abnormalities found in the offspring of influenza-infected or poly(l:C)-treated dams. Moreover, co-injection of an anti-IL-6 antibody blocks the effects of poly(I:C), yielding offspring with normal behavior. The critical

\footnotetext{
${ }^{1}$ Department of Psychiatry, Vanderbilt University, Nashville, TN, USA; ${ }^{2}$ Division of Biology, California Institute of Technology, Pasadena, CA, USA; ${ }^{3}$ Department of Psychiatry, University of Szeged, Szeged, Hungary and ${ }^{4}$ Vanderbilt Kennedy Center for Research on Human Development, Vanderbilt University, Nashville, TN, USA Correspondence: Dr K Mirnics, Department of Psychiatry, Vanderbilt University, 8130A MRB III, 465 21st Avenue South, Nashville, TN 37203 , USA.

E-mail: karoly.mirnics@vanderbilt.edu

${ }^{5}$ These two authors contributed equally to this work.

Keywords: autism; brain; crystallin; gene expression; maternal immune activation; schizophrenia

Received 09 November 2011; revised 27 February 2012; accepted 03 March 2012
} 
importance of IL-6 in MIA is further shown by the finding that poly(I:C) injection in IL-6 knockout mice also yields offspring with normal behavior. ${ }^{17}$ Flavonoids that block JAK/STAT signaling downstream of the IL-6 receptor also block the induction of abnormal behaviors by maternal IL- 6 injection. ${ }^{18}$ Maternal IL- 6 is also critical for the endocrine changes in the placenta induced by MIA. ${ }^{19,20}$

However, the immediate effects of MIA on the fetal brain are not well understood. It is critical to examine the very early effects because the rise in IL- 6 caused by maternal IL- 6 injection is transient, as is the effect of injecting the anti-IL-6 blocking antibody in the pregnant dam. These acute effects nonetheless lead to permanent changes in the behavior of the offspring. To gain a better understanding of the molecular events that take place in the MIA-exposed developing brain, we examined the transcriptome changes associated with three different MIA models, and identify the critical early mediators that may contribute to the emergence of behavioral symptoms in MIA offspring.

\section{Materials and methods}

Animals and MIA treatments. All procedures involving animals were approved by the Caltech Animal Care and Use Committee. Female C57BL/6J mice (The Jackson Laboratory, Bar Harbor, ME, USA) were obtained from our in-house breeding facility and were housed in ventilated cages under standard laboratory conditions. Mice were mated overnight, and the presence of a vaginal plug marked that day as embryonic day 0 (E0). Pregnant females were not disturbed, except for weekly cage cleaning, until E9.5 when they were weighed and pseudo-randomly assigned to one of four groups. Each group contained four to five pregnant females.

Treatments with human influenza virus, poly $(\mathrm{I}: \mathrm{C})$ and recombinant IL-6 (rIL-6) were used to induce MIA at conditions that previously resulted in offspring altered behavior. ${ }^{10,17}$ To allow for time to develop a flu infection, pregnant mice on E9.5 were anesthetized intraperitoneally with $10 \mathrm{mg} \mathrm{kg}^{-1}$ xylazine and $100 \mathrm{mg} \mathrm{kg}^{-1}$ ketamine and inoculated intranasally with 6000 plaque-forming units of human influenza virus in $90 \mu \mathrm{l}$ phosphate-buffered saline (PBS). Pregnant mice on E12.5 were injected with saline, poly $(\mathrm{I}: \mathrm{C})$ or rlL-6. For poly(l:C) injections, poly(l:C) potassium salt (Sigma Aldrich, St Louis, MO, USA) was freshly dissolved in saline and administered intraperitoneally at $20 \mathrm{mg} \mathrm{kg}^{-1}$ based on the weight of the poly $(\mathrm{I}: \mathrm{C})$ itself, not including the total weight of the potassium salts. Control mice were injected with saline alone at $5 \mu \mathrm{g} \mathrm{g}^{-1}$ body weight. For rlL-6 injections, $5 \mu \mathrm{g}$ carrierfree, mouse rlL-6 (eBioscience, San Diego, CA, USA) was freshly dissolved in $150 \mu \mathrm{l}$ saline and injected intraperitoneally. Pregnant mice were killed on E12.5 (3 h after poly(l:C) or rIL-6 injection and 3 days after influenza inoculation), and the embryonic brains were removed quickly and frozen in $1.5 \mathrm{ml}$ tubes.

Transcriptome analysis. RNA was isolated from individual embryonic brains using PureLink RNA mini kit (Ambion, Chicago, IL, USA). RNA quality was assessed using the Agilent (Palo Alto, CA, USA) Bioanalyzer. To reduce gene expression variability that was not related to MIA exposure, RNA from each embryonic brain within a litter was pooled into a single sample, and used for the generation of biotinlabeled sample for microarray hybridization (Supplemental Material 1). This allowed us to identify MIA-induced gene expression changes that were commonly found in the embryonic brains of each litter, resulting in 4-5 replicates for each of the four conditions. Thus, from a total of 138 embryos, 17 pooled RNA samples (each originating from all the embryonic brains within an individual pregnant dam) were generated and hybridized on a GeneChip HT MG-430 PM 24-Array Plate. On the basis of the variability in our previous mouse experiments, ${ }^{21}$ we estimated that we had an $80 \%$ probability to identify significant gene expression changes that were $>30 \%$. The data were log 2 transformed using the RMA algorithm, and differential expression was established. A gene was considered differentially expressed if (1) the average $\log 2$ ratio (ALR) between the experimental treatment and control exceeded $30 \%(|A L R|>0.3785)$ and (2) the statistical significance of differences was $P<0.05$ using the Student's $t$-test. The GenePattern software (Broad Institute, Cambridge, CA, USA) was used for hierarchical clustering of the gene expression intensities.

Quantitative PCR. RNA isolated from embryos residing in the left or the right uterine horn was pooled proportionately, so there were two samples per each pregnant dam. Individual embryonic brain RNA or pooled RNA samples were used to generate cDNA with high-capacity cDNA reverse transcription kit (Applied Biosystems, San Mateo, CA, USA). SYBR Green intercalation quantitative PCR (qPCR) was run in a 7300 real-time PCR system (Applied Biosystems) with the following primers: crystallin $\gamma A$ (cryaa) (F: 5'-TCTT CTTGGACGTGAAGCAC-3', R: 5'-GAAATGTAGCCATGGT CATCC-3'), crystallin $\alpha B$ (cryab) (F: 5'-TGTGAATCTGGA CGTGAAGC-3', R: 5'-TGACAGGGATGAAGTGATGG-3'), crystallin $\beta A 1$ (cryba1) (F: 5'-CCTGGAAAGAGGAGAATA CCC-3', R: 5'-TTATGATTAGCGGAACAGATGG-3'), crystallin BA2 (cryba2) (F: 5'-ACCAGCAAAGATGTGGGTTC-3', R: 5'-GCCTAATGCTGGACTCTTCG-3'), crystallin $\beta A 4$ (cryba4) (F: 5'-CACCACTCAGGTGACTACAAGC-3', R: 5'-CCAGA GGACACAAGGGTAGC-3'), crystallin $\beta B 1$ (crybb1) (F: 5'-TCCCAGGAACATAAGATCTGC-3', R: 5'-ACGGTCACA GAAGCCATAAAC-3'), crystallin $\beta B 3$ (crybb3) (F: 5'-CAG CCGACGTAGTGACATTC-3', R: 5'-TCATCTACGATCTCC ATCTTGC-3'), crystallin $\gamma \mathrm{B} / \mathrm{A} / \mathrm{C}$ (crygb/a/c) (F: 5'-AGCG AGATGGGAAAGATCAC-3', R: 5'-AGTACTGGTGGCCCT GGTAG-3') and crystallin $\gamma$ C/A (crygc/a) (F: 5'-TGCGGCT GTATGAGAAAGAA-3', R: 5'-CCTCGGTAGTTAGGCATC TCA-3').

All of these custom-designed primers reported amplification efficiency $>90 \%$. qPCR data were analyzed using the ddCt method with the housekeeping gene phosphoglycerate kinase 1 (PGK1) as a normalizer.

In addition, qPCR for the crystallin genes cryaa, cryba1 and $c r y b b 1$ was performed with 34 RNA samples pooled from the embryos identified as residing in the left or right uterine horns. Furthermore, the expression of cryaa, cryba 1 and crybb1 was also examined by qPCR in all 36 individual embryonic brains originating from the influenza-treated dams. 
Correlation of placental weight with gene expression measurements. At the time of the dissection, the weight of the individual placentas was measured for each embryo. Placental weight was assessed across the treatments with a groupwise, two-tailed $t$-test. In addition, qPCR-reported crystallin expression was correlated with average placental weight across the treatments and control groups using Pearson correlation.

\section{Results}

Transcriptome changes in flu-, poly(I:C)- and rIL-6treated embryonic brains. Five pregnant dams were treated with a single, nasal instillation of flu virus at E9.5. The embryos were dissected on E12.5, the time of peak maternal IL-6 expression, and brains were collected. A single treatment of poly(l:C), rlL-6 or saline was administered to four pregnant dams per group at E12.5. The embryonic brains from these treatments were collected $3 \mathrm{~h}$ after injection. RNA was isolated from the entire brain of individual embryos. We obtained 31 to 36 embryos per treatment, which totaled 138 embryonic brain samples. For microarray analysis, embryonic brains from each litter were pooled into a single sample. Using a $30 \%$ expression difference from saline-injected controls and a statistical significance of $P<0.05$, we identified 256 genes differentially expressed in the flu model, 294 in the poly(I:C) model and 195 in the IL-6 model. Examining all of the differentially expressed genes in a two-way (samples and genes) unsupervised hierarchical clustering of the gene expression intensities, the four experimental groups separate into distinct clusters (Figure 1a) that correspond to the different MIA treatments, with the main clusters separating the saline treatment from all three MIA treatments. Interestingly, the majority of the genes altered in the poly(I:C) and IL-6 treatments were upregulated, although there were as many downregulated genes in the flu treatment, which might reflect secondary expression changes that occurred during the 3day period following the influenza virus exposure.

Next, we tested if the transcriptome changes showed a common expression pattern across the three different MIA treatments. We created three groups of the most differentially expressed genes: 256 genes in the flu model, 294 in the poly(I:C) model and 195 in the IL- 6 model. The mRNA signature of the 256 differentially expressed transcripts in the flu model was assessed in the poly(I:C) and rIL-6 transcriptomes. We found that, as a group, the flu model transcripts are also significantly changed in the poly $(\mathrm{I}: \mathrm{C})$ and IL-6 models for MIA $(r=0.88, P=1.8 \mathrm{E}-84$ and $r=0.85, P=5.95 \mathrm{E}-72$, respectively) (Figures $1 \mathrm{~b}$ and $\mathrm{c}$ ). Likewise, the 195 differentially expressed genes in the IL-6-MIA group show a significant expression difference in the flu-model and poly(l:C)-model brains ( $r=0.85, P=1.42 \mathrm{E}-55$ and $r=0.85$, $P=1.06 \mathrm{E}-54$, respectively) (Figures $1 \mathrm{~d}$ and e), and the 294 transcript changes in the IL-6 group are also significantly present in the flu and poly $(\mathrm{I}: \mathrm{C})$ treatments $(r=0.59$, $P=8.78 \mathrm{E}-29$ and $r=0.59, \quad P=3.9 \mathrm{E}-29$, respectively) (Figures $1 \mathrm{f}$ and $\mathrm{g}$ ). This is a strong indication that all three treatments affect similar molecular processes, although their effects might be somewhat different because of the timing difference in flu exposure and potential downstream signaling differences. A comprehensive list of the differentially expressed genes and their expression levels is provided in Supplemental Material 2.

Of the transcripts with 'most changed' expression ( $>30 \%$ expression change and $P<0.05$ in all three treatments), 41 genes were altered in both IL- 6 and flu treatment, 31 in poly(l:C) and flu, 17 in poly(I:C) and IL-6 and 12 genes were similarly changed in all three MIA models. A hierarchical clustering of the expression intensities of these 12 genes clearly separates the saline-treated controls from the flu, poly(I:C) and IL-6 treatments (Figure 2).

\section{Common gene expression changes among treatments point} to involvement of the crystallin gene family. Surprisingly, 5 of the 12 genes that are upregulated in all three MIA treatments belong to the crystallin gene family: cryaa, crybb3, $c r y b b 1$, cryba1 and crygb/crygc. To validate this finding with an independent method, we generated cDNA from pooled RNA from embryos in each dam. To inquire about a possible effect of location in the uterus, RNA was separately pooled from embryos residing in the left and the right uterine horns, so there were two samples for each dam. For more detailed view on the crystallin family of genes, we also tested the expression of cryab, cryba2, cryba4 and crygc/a. The qPCR data are highly correlated with the microarray data (flu: $r=0.82, P=$ 0.007 ; poly(l:C): $r=0.84, P<0.005$; rlL-6: $r=0.89, P=0.001$ ) (Figure 3 ). No obvious effect of position is apparent.

As the expression of these crystallin genes is highly coordinated, we selected only cryaa, cryba1 and crybb1 for further qPCR analysis using RNA from the individual fluexposed embryonic brains. The upregulation of crystallin transcripts observed by microarray is confirmed by the qPCR analysis of the 36 individual embryonic RNA samples. Flu treatment results in significantly increased cryaa, cryba1 and $c r y b b 1$ transcript levels (cryaa: $\mathrm{ddCt}=1.97, P=0.001$; cryba1: $\operatorname{ddCt}=1.39, \quad P=0.01 ; \quad$ crybb1: $\quad \mathrm{ddCt}=2.00, \quad P=$ 0.0002) (Figure 4). However, these data also reveal a remarkable individual variability in flu-induced crystallin upregulation, which does not correspond to the uterine position of the embryos (flu: s.d. $=2.8$; saline: s.d. $=1.6$ ). This indicates that flu exposure does not equally affect all exposed embryonic brains within each dam, which could potentially contribute to eventual behavioral variability.

Crystallin family upregulation is transient. As the crystallin family genes are significantly upregulated at both $3 \mathrm{~h}$ following $\mathrm{IL}-6$ and poly $(\mathrm{I}: \mathrm{C})$ treatment and at 3 days following flu treatment, we asked if these expression changes persist throughout the lifespan of the offspring. We examined the expression of cryaa, cryba1 and crybb1 in the frontal cortex and hippocampus of 15-week-old pups $(n=12)$ from poly(l:C)- and saline-treated dams. We find no significant difference between these groups in the expression of these genes. Furthermore, no difference is found in the frontal cortex between 6-month-old $(n=6)$ offspring of saline-, rIL-6- and poly(I:C)-treated dams. Similarly, in a different cohort of mice, no difference in crystallin expression is seen in the frontal cortex and hippocampus of poly(l:C)- and 
a

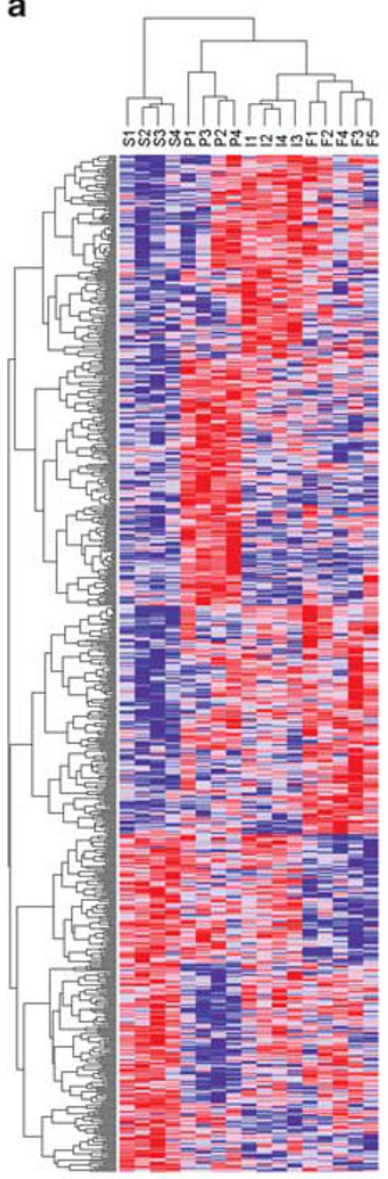

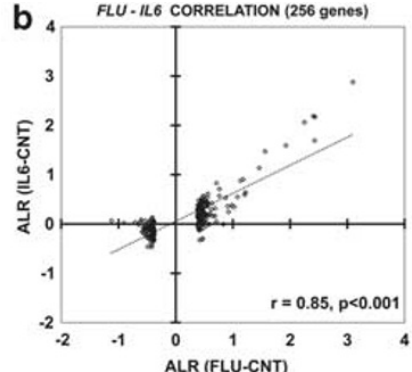
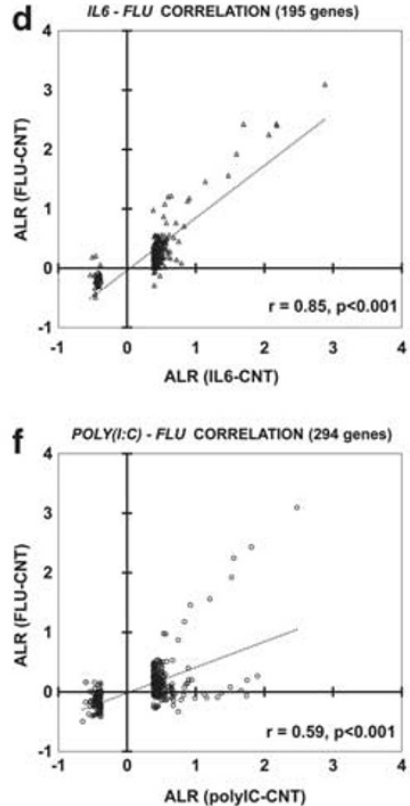
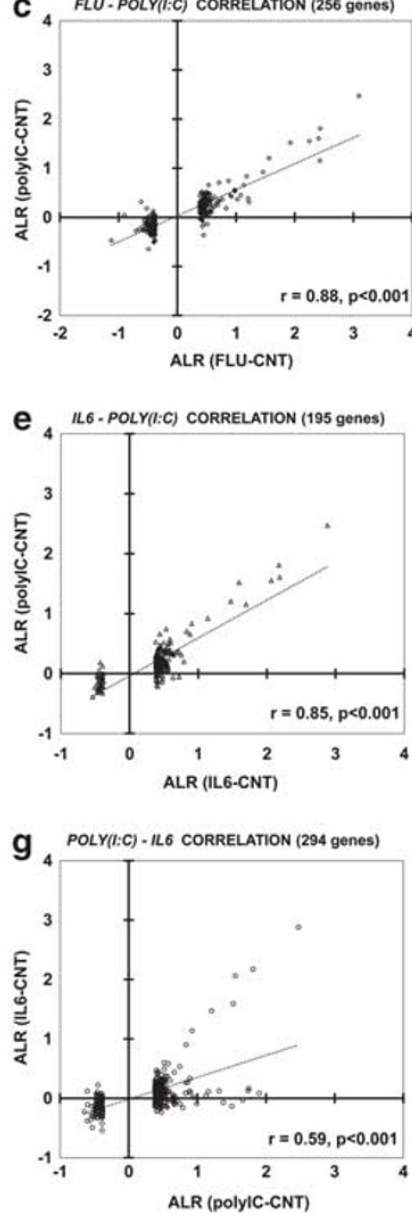

Figure 1 The transcriptome of the embryonic brain is altered in three maternal immune activation (MIA) models. (a) All differentially expressed transcripts in the MIA treatments were subjected to a two-way (horizontal: genes; vertical: samples) unsupervised clustering using GenePattern. Gene expression differences are color-coded (redincreased expression; blue-decreased expression), and the intensity of the color corresponds to the magnitude of gene expression change. Note that the experimental groups separate into distinct clusters that correspond to the different MIA treatments, with the main clusters separating the saline treatment from all three MIA treatments. (b-g) Three data set-driven gene groups were created, each containing genes that were differentially expressed in one of the three MIA models (256 genes in the flu model, 294 in the poly(I:C) model and 195 in the recombinant IL-6 (rlL-6) model). We then examined the expression of each of these gene groups in the other two MIA cohorts. Thus, the pattern of differentially expressed genes in the flu treatment was tested in the poly $(\mathrm{l}: \mathrm{C})$ and $\mathrm{rlL}-6$ cohorts $(\mathbf{b}$ and $\mathbf{c}$, respectively), the pattern of differentially expressed genes in the rlL-6 treatment was tested in the flu and poly $(\mathrm{I}: \mathrm{C})$ cohorts (d and $\mathbf{e}$, respectively) and the pattern of differentially expressed genes in the poly(l:C) treatment was assessed in the flu and rIL-6 cohorts (f and $\mathbf{g}$, respectively). Note that the gene expression patterns uncovered in any of the three MIA treatments show a very strong, highly significant correlation to the other two MIA treatments.

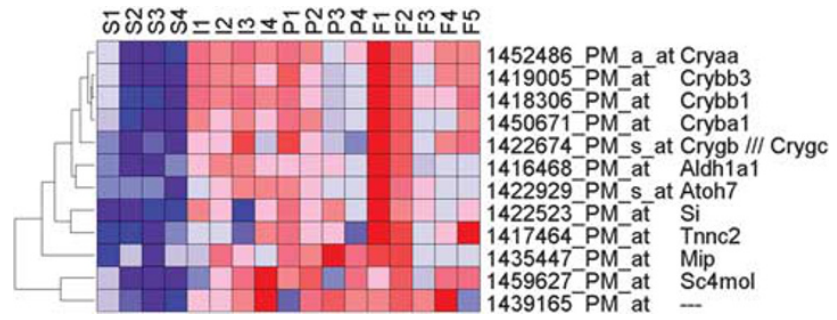

Figure 2 The most significantly changed transcripts in all three maternal immune activation (MIA) treatments include multiple members of the crystallin gene family. Clustering was performed as described in Figure 1a. Note that, of the 12 gene expression changes observed in all three MIA conditions, 5 are mRNA species belonging to the crystallin gene family.

saline-treated dams $(n=15)$ at 12 months of age. Thus, we conclude that the upregulation of crystallin genes observed across the three MIA models is transient.
Crystallin expression level correlates with placental weight. MIA has a strong effect on development, and at least part of this effect is mediated through the influence of maternal IL- 6 on the placenta. ${ }^{19,20}$ To test whether there is a relationship between the effects of MIA on the placenta and on the fetal brain, we measured the placental weight of individual embryos from the three MIA conditions (Figure 5). The average placental weight is significantly decreased in all three MIA treatments compared to saline treatment (flu: $P=0.00016$; poly(I:C): $P=0.03439$; IL-6: $P=0.0041$ ). Furthermore, brain expression levels of cryaa, cryba1 and $c r y b b 1$ determined by qPCR are highly correlated with the decrease in the average placental weight obtained for all conditions (cryaa: $r=0.85, P=0.072$; cryba1: $r=0.72$, $P=0.138$; crybb1: $r=0.92, P=0.041$, respectively). Thus, the response of the crystallin genes corresponds closely with the degree of MIA. 


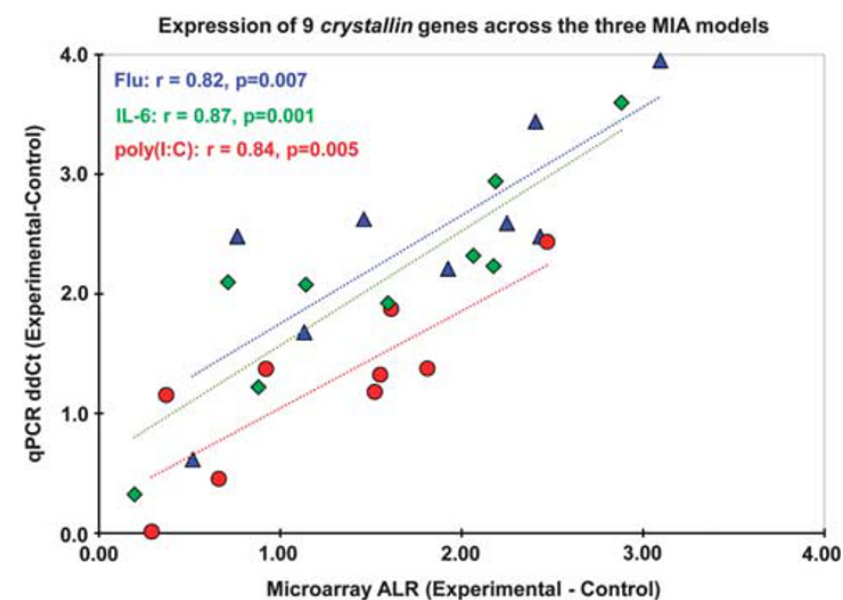

Figure 3 Quantitative PCR (qPCR) validates crystallin gene expression changes across the maternal immune activation (MIA) models. The expression of nine genes was tested (crystallin $\alpha \mathrm{A}$ (cryaa), crystallin $\gamma \mathrm{B} / \mathrm{C} / \mathrm{A}$ (crygb/c/a), crystallin $\gamma \mathrm{C} / \mathrm{A}$ (crygc/a), crystallin $\beta \mathrm{B} 1$ (crybb1), crystallin $\beta \mathrm{A} 2$ (cryba2), crystallin $\beta \mathrm{B} 3$ (crybb3), crystallin $\beta A 1$ (cryba1), crystallin $\beta A 4$ (cryba4), crystallin $\alpha \mathrm{B}$ (cryab)) on pools of RNA derived from the three MIA treatments. The $X$ axis denotes average $\log 2$ ratios reported by microarrays, whereas the ddCt $q P C R$ values are plotted on the $Y$ axis. Note that the qPCR and microarray-reported expression changes are highly correlated across the three MIA models.

\section{Crystallin expression in Flu-MIA}

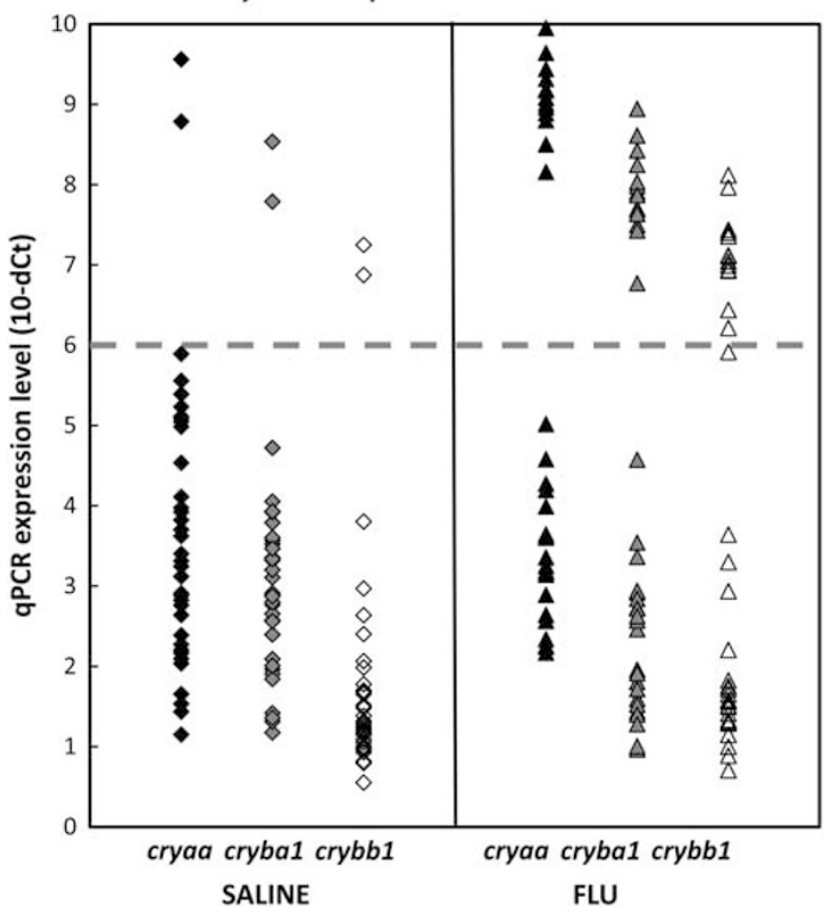

Figure 4 Crystallin expression is variable in individual brains from flu-exposed embryos. Expression of crystallin $\alpha \mathrm{A}$ (cryaa), crystallin $\beta \mathrm{A} 1$ (cryba1) and crybb1 was assessed by quantitative PCR (qPCR) across the individual brains of 36 flu and 36 saline embryos. Each symbol represents a phosphoglycerate kinase 1 (PGK1)normalized gene expression level in a single embryonic brain. The dashed gray line denotes the reliable transcript detection threshold by qPCR. Note the upregulation of the three crystallin genes in the flu group compared with controls, and the considerable variability of crystallin expression levels between the flu-exposed embryonic brains.

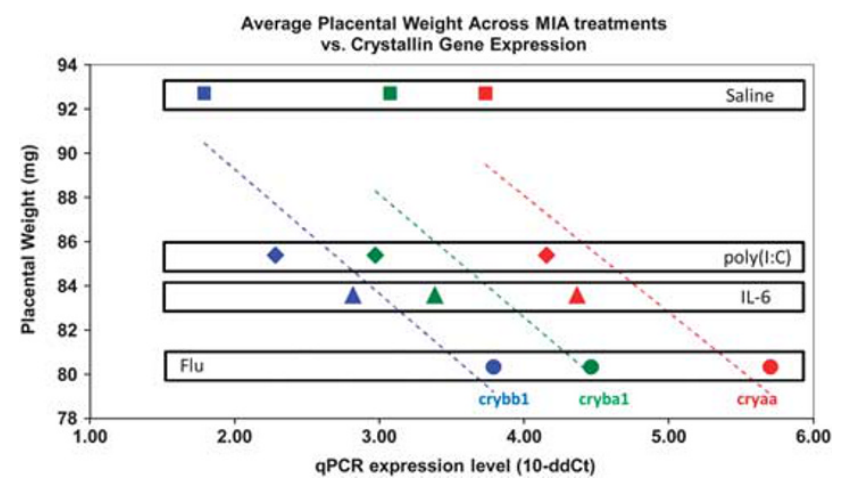

Figure 5 Crystallin expression is correlated with placental weight. quantitative PCR (qPCR) expression levels of cryaa (red), cryba1 (green) and crybb1 (blue) are correlated with mean placental weights across the maternal immune activation (MIA) groups and controls. The $X$ axis denotes the magnitude of expression change measured in $\mathrm{ddCT}(1 \mathrm{Ct}=2$-fold change) and the $Y$ axis depicts average placental weight. Squares denote saline treatment, diamonds denote poly $(\mathrm{I}: \mathrm{C})$ treatment, triangles denote IL- 6 treatment and circles denote flu treatment. Note that the average placental weight is significantly different between the controls and all three MIA treatments, and that the magnitude of all the crystallin expression changes is highly correlated with placental weight.

\section{Discussion}

This study of the acute effects of MIA on the embryonic brain transcriptome reveals that: (1) all three MIA treatments (flu, poly(I:C) and IL-6) evoke strong gene expression changes in the embryonic brain; (2) the three MIA treatments yield diverse as well as overlapping transcriptome signatures; (3) all MIA treatments strongly upregulate the crystallin gene family, represented by $\alpha, \beta$ and $\gamma$ members, in the embryonic brain; (4) the upregulation of crystallin genes is an acute reaction that does not persist into adulthood; and (5) the level of crystallin gene expression is correlated with the degree of MIA as measured by placental weight. In addition, our data also reveal a remarkable variability in crystallin induction among the individual brains in the flu MIA model, indicating that this form of MIA does not affect all of the embryos equally within each dam.

Although we find that the three MIA models induce a number of genes in common, there are also significant differences among the groups. This can be attributed in part to differences in the molecular action of the three perturbation agents, and also to the differences in the time point of the flu, poly(I:C) and IL-6 exposure. The efficiency of flu exposure and disease time course is challenging to control, and the flu-triggered cytokine response is gradual and takes days to develop. The time point chosen for IL-6 and poly(I:C) injection (E12.5) approximately mimics the peak of IL-6 induction following flu infection (E9.5). ${ }^{10,17}$ However, cytokine induction is longer lasting following infection than it is for the other two treatments. Likewise, the molecular effects of poly $(\mathrm{I}: \mathrm{C})$ versus IL-6 at $3 \mathrm{~h}$ after exposure may not be fully equivalent, resulting in both common and divergent gene expression changes at the same time point. Thus, we believe that the overall data argue that the transcriptome differences we observe are part of the same process, providing molecular 'snapshots' of canonical, transient expression changes that are rapidly 
changing. Nonetheless, the common gene expression patterns across the three MIA models likely give rise to the behavioral deficits that are common in the offspring of all three treatments such as prepulse inhibition, social interaction and open-field exploration. ${ }^{10,17}$ This possibility provides strong motivation to further pursue the potential effects of dysregulation of these particular genes on brain development, with a primary focus on the role of the crystallin gene family.

That elevated brain crystallin expression is common among three MIA mouse models aligns well with studies implicating crystallins in human autism and schizophrenia. Altered $\alpha \mathrm{B}$ crystallin protein levels are detected in the frontal cortex of autistic brains. ${ }^{22}$ In addition, anti- $\alpha \mathrm{B}$ crystallin antibodies are reported to be elevated in the sera of autistic subjects compared with controls. ${ }^{23}$ Moreover, increased expression of $\alpha \mathrm{B}$-crystallin is detected in Rett syndrome brains, ${ }^{24}$ and $\alpha B$-crystallin is found in inclusions in brains from patients with Fragile $\mathrm{X}$-associated tremor/ataxia syndrome. ${ }^{25}$ In schizophrenia, altered expression of both $\alpha$-crystallin and $\mu$-crystallin are reported in the anterior cingulate cortex ${ }^{26}$ and prefrontal cortex. ${ }^{27,28}$ Furthermore, dysregulated brain crystallin levels are implicated in the manifestation of a variety of neurodegenerative disorders, including Parkinson's disease, Alzheimer's disease, multiple sclerosis, Creutzfeldt-Jakob disease and Alexander disease. ${ }^{29-35}$

Although the exact role of crystallins in these disorders is unclear, studies in animal models suggest that upregulated crystallin levels serve a neuroprotective function. Knocking out $\alpha \mathrm{B}$-crystallin in Alzheimer's disease or experimental autoimmune encephalitis mice causes worsened symptoms. ${ }^{36,37}$ These effects in experimental autoimmune encephalitis mice are in turn ameliorated by systemic delivery of recombinant $\alpha \mathrm{B}$-crystallin, ${ }^{37}$ as are the effects of experimental stroke in mice. ${ }^{38}$ Similarly, transgenic overexpression of $\alpha \mathrm{B}$-crystallin ameliorates symptoms in a mouse model of Alexander disease, a fatal developmental neurodegenerative disorder originating in astrocytes. ${ }^{39}$ In addition, $\alpha \mathrm{A}$-crystallin exhibits anti-apoptotic protective effects in experimentally induced inflammation of the uvea, ${ }^{40}$ and pretreatment with $\alpha$-crystallin blocks systemically induced inflammation in the brain. $^{41}$

Recent studies have shown that, in addition to their canonical roles as molecular chaperones, crystallins serve a wide variety of other functions that may underlie their protective roles in vivo. For example, $\alpha \mathrm{A}$-crystallin-mediated inhibition of caspase-3 rescues Pax6-deficient dopaminergic neurons from apoptosis. ${ }^{42}$ Likewise, $\alpha \mathrm{B}$-crystallin protects against Fas/APO-1-induced cell death in vitro. Crystallins can also confer neuroprotection by modulating cytoplasmic calcium levels. Retinal $\beta$ B2-crystallin, for example, colocalizes with synaptotagmin 1, a primary calcium sensor, as well as calmodulin, a major calcium-binding protein, ${ }^{43}$ and several crystallins harbor Greek key motifs that sequester calcium ions. ${ }^{44-46}$ These properties in $\alpha$ - and $\beta B 2$-crystallins are thought to confer their ability to promote neurite outgrowth during retinal repair. ${ }^{43,47}$ Another pathway of crystallin activity is through the regulation of oxidative stress and acute phase immune responses. For instance, $\alpha B$-crystallin modulates nuclear factor- $\kappa \mathrm{B}$ activity based on its phosphorylation status, ${ }^{48}$ and pretreatment with $\alpha \mathrm{B}$-crystallin prevents nuclear factor- $\kappa \mathrm{B}$-induced neurotoxic effects. ${ }^{49}$ Moreover, $\alpha \mathrm{B}$-crystallin administration in vitro and in vivo downregulates tumor necrosis factor- $\alpha$ and nitric oxide synthase in activated microglia. ${ }^{50}$

Given this literature, the induction of crystallins in response to MIA can be seen as an attempt of the developing brain to counteract the immune system challenge. Importantly, the crystallin upregulation we observe in embryos in response to MIA cannot be detected in the hippocampus and the frontal cortex of MIA-treated adolescent mice, indicating that crystallin induction is transient and characteristic of the acute phase of the MIA exposure. Furthermore, comparing our data to previous transcriptome studies of MIA offspring suggests that the fetal and adolescent/adult brain transcriptome changes do not share significant commonalities. ${ }^{17,51,52}$

In addition to crystallin upregulation, several other genes are altered in all three models of MIA tested here. For some of them (mip, si, tnnc2), there is insufficient knowledge about the function of the proteins they encode, which makes it difficult to envision their particular roles in the brain, while other transcripts are likely to play an important role in brain development. Aldh1a1 is an important player in environmentally induced oxidative damage ${ }^{53}$ and this might explain its strong upregulation in these MIA models. As an enzyme, ALDH1A1 exerts a cellular protective role by metabolizing highly reactive aldehydes, including ethanol metabolite acetaldehyde and major products of lipid peroxidation. ALDH1A1 is best known for converting retinaldehyde to retinoic acid, and altered retinoic acid levels in the brain might disrupt normal anteroposterior neural development. ${ }^{54}$ Similarly, sterol-C4-methyl oxidase-like protein (SC4MOL) is localized to the endoplasmic reticulum membrane and is believed to be involved in cholesterol biosynthesis, which when dysregulated has clinical manifestations in neurological development. ${ }^{55}$ In addition, atonal homolog 7 (atoh7) is involved in the development of the optic nerve. ${ }^{56}$ Therefore, the upregulation of these three genes, aldh1a1, sc4mol and atoh7, may represent an important change in the molecular environment of the embryonic brain that could result in aberrant neural development. Finally, we find that the expression levels of many genes with known roles in cell cycle regulation, neuronal development and differentiation (for example, cyclin-dependent kinase 12, cyclin-dependent kinase 17, insulin-like growth factor binding protein 3 , neural cell adhesion molecule 1 and 2 , transforming growth factor $\beta 2$, transducer of ERBB2, 2, and so on) are highly correlated with the expression of the crystallins (Supplemental Material 2).

The common gene expression pattern across the various MIA treatments, in conjunction with previous findings, argues that IL-6 is a critical mediator of MIA, and that crystallins are important molecular mediators of this process. This raises several important questions. First, is the IL-6/crystallin cascade a promising target for molecular intervention in the context of schizophrenia and autism treatment or prevention? Second, are the currently used anti-inflammatory therapies (which show promise as adjuvant therapy in schizophrenia) modulating the IL-6/crystallin pathway, and acting through this mechanism? Third, how is crystallin overexpression in the embryonic brain disrupting normal development, and does it alter the typical connectivity between the developing brain 
regions? Finally, what is the critical time window for modulating the IL-6/crystallin pathway during which we can reverse the detrimental behavioral effects of MIA? The answers to these critical questions remain mostly unknown to date, and will have to be answered by comprehensive follow-up experiments.

Overall, based on our findings and the known molecular effects of crystallins, we propose that the induction of the crystallin genes in response to MIA is a neuroprotective attempt of the developing brain to counteract environmental stress. However, this response is likely to have detrimental consequences. Owing to their additional roles in neuronal differentiation and axonal growth, ${ }^{42,43}$ overexpression of crystallins (and other genes such as aldh1a1, sc4mol and atoh7) might tip the delicate balance between neurogenesis and differentiation in the embryonic brain. This view is strongly supported by the observation that poly(I:C) MIA leads to decreased hippocampal and cortical neurogenesis. ${ }^{57,58} \mathrm{We}$ also propose that once the immune activation disappears, the normal brain developmental program resumes, but with a time lag. As a result, layer formation in the cortex ${ }^{59}$ and communication between the various brain regions is altered, leading to permanent changes in connectivity and neurochemistry. ${ }^{60}$ This ultimately results in the various behavioral abnormalities that are found in the offspring of all three MIA models. Finally, we also argue that this cascade of events might parallel the mechanisms by which environmental insults contribute to the risk of neurodevelopmental disorders such as schizophrenia and autism.

\section{Conflict of interest}

The authors declare no conflict of interest.

Acknowledgements. We are thankful for the microarray work performed by the Genome Sciences Resource at Vanderbilt University and for valuable comments of the manuscript provided by Martin J Schmidt. The experiments were supported by NIH R01 MH067234 (KM), MH079299 (KM), Cure Autism Now (PHP), McKnight Foundation Neuroscience of Brain Disorder Award (PHP), NIH RO1 MH067978 (PHP), Autism Speaks Dennis Weatherstone Pre-Doctoral Fellowship (EYH) and NRSA 5 T32GM07737 (EYH).

1. Brown AS, Derkits EJ. Prenatal infection and schizophrenia: a review of epidemiologic and translational studies. Am J Psychiatry 2010; 167: 261-280.

2. Atladottir $\mathrm{HO}$, Thorsen $\mathrm{P}$, Ostergaard L, Schendel DE, Lemcke $\mathrm{S}$, Abdallah $\mathrm{M}$ et al. Maternal infection requiring hospitalization during pregnancy and autism spectrum disorders. J Autism Dev Disord 2010; 40: 1423-1430.

3. Abdallah MW, Larsen N, Grove J, Norgaard-Pedersen B, Thorsen P, Mortensen EL et al. Amniotic fluid chemokines and autism spectrum disorders: an exploratory study utilizing a Danish Historic Birth Cohort. Brain Behav Immun 2012; 26: 170-176.

4. Goines PE, Croen LA, Braunschweig D, Yoshida CK, Grether J, Hansen R et al. Increased midgestational IFN-gamma, IL-4 and IL-5 in women bearing a child with autism: a casecontrol study. Mol Autism 2011; 2: 13

5. Rosenberg RE, Law JK, Yenokyan G, McGready J, Kaufmann WE, Law PA. Characteristics and concordance of autism spectrum disorders among 277 twin pairs Arch Pediatr Adolesc Med 2009; 163: 907-914.

6. Hallmayer J, Cleveland S, Torres A, Phillips J, Cohen B, Torigoe T et al. Genetic heritability and shared environmental factors among twin pairs with autism. Arch Gen Psychiatry 2011; 68: 1095-1102.

7. Patterson PH. Immune involvement in schizophrenia and autism: etiology, pathology and animal models. Behav Brain Res 2009; 204: 313-321.

8. Fatemi SH, Emamian ES, Kist D, Sidwell RW, Nakajima K, Akhter P et al. Defective corticogenesis and reduction in Reelin immunoreactivity in cortex and hippocampus of prenatally infected neonatal mice. Mol Psychiatry 1999; 4: 145-154.
9. Fatemi SH, Cuadra AE, El-Fakahany EE, Sidwell RW, Thuras P. Prenatal viral infection causes alterations in nNOS expression in developing mouse brains. NeuroReport 2000; 11: 1493-1496.

10. Shi L, Fatemi SH, Sidwell RW, Patterson PH. Maternal influenza infection causes marked behavioral and pharmacological changes in the offspring. J Neurosci 2003; 23: 297-302.

11. Palmen SJ, van Engeland $H$, Hof $P R$, Schmitz $C$. Neuropathological findings in autism. Brain 2004; 127(Part 12): 2572-2583.

12. Amaral DG, Schumann CM, Nordahl CW. Neuroanatomy of autism. Trends Neurosci 2008; 31: $137-145$.

13. Moreno JL, Kurita M, Holloway T, Lopez J, Cadagan R, Martinez-Sobrido L et al. Maternal influenza viral infection causes schizophrenia-like alterations of 5-HTA and mGlu receptors in the adult offspring. J Neurosci 2011; 31: 1863-1872.

14. Meyer U, Feldon J. Epidemiology-driven neurodevelopmental animal models of schizophrenia. Prog Neurobiol 2010; 90: 285-326.

15. Patterson $\mathrm{PH}$. Maternal infection and immune involvement in autism. Trends Mol Med 2011; 17: 389-394

16. Meyer U, Nyffeler M, Schwendener S, Knuesel I, Yee BK, Feldon J. Relative prenatal and postnatal maternal contributions to schizophrenia-related neurochemical dysfunction after in utero immune challenge. Neuropsychopharmacology 2008; 33: 441-456.

17. Smith SE, Li J, Garbett K, Mirnics K, Patterson PH. Maternal immune activation alters fetal brain development through interleukin-6. J Neurosci 2007; 27: 10695-10702.

18. Parker-Athill E, Luo D, Bailey A, Giunta B, Tian J, Shytle RD et al. Flavonoids, a prenatal prophylaxis via targeting JAK2/STAT3 signaling to oppose IL-6/MIA associated autism. $\checkmark$ Neuroimmunol 2009; 217: 20-27.

19. Hsiao EY, Patterson PH. Activation of the maternal immune system induces endocrine changes in the placenta via IL-6. Brain Behav Immun 2011; 25: 604-615.

20. Mandal M, Marzouk AC, Donnelly R, Ponzio NM. Maternal immune stimulation during pregnancy affects adaptive immunity in offspring to promote development of $\mathrm{TH} 17$ cells. Brain Behav Immun 2010; 25: 863-871.

21. Glorioso C, Sabatini M, Unger T, Hashimoto T, Monteggia LM, Lewis DA et al. Specificity and timing of neocortical transcriptome changes in response to BDNF gene ablation during embryogenesis or adulthood. Mol Psychiatry 2006; 11: 633-648.

22. Pickett J. Current investigations in autism brain tissue research. J Autism Dev Disord 2001; 31: $521-527$

23. Vojdani A, Campbell AW, Anyanwu E, Kashanian A, Bock K, Vojdani E. Antibodies to neuron-specific antigens in children with autism: possible cross-reaction with encephalitogenic proteins from milk, Chlamydia pneumoniae and Streptococcus group A. J Neuroimmunol 2002; 129: 168-177.

24. Colantuoni $\mathrm{C}$, Jeon $\mathrm{OH}$, Hyder K, Chenchik A, Khimani AH, Narayanan V et al. Gene expression profiling in postmortem Rett syndrome brain: differential gene expression and patient classification. Neurobiol Dis 2001; 8: 847-865.

25. Iwahashi CK, Yasui DH, An HJ, Greco CM, Tassone F, Nannen $\mathrm{K}$ et al. Protein composition of the intranuclear inclusions of FXTAS. Brain 2006; 129(Part 1): 256-271.

26. Martins-de-Souza D, Schmitt A, Roder R, Lebar M, Schneider-Axmann T, Falkai P et al. Sex-specific proteome differences in the anterior cingulate cortex of schizophrenia. $J$ Psychiatr Res 2010; 44: 989-991.

27. Arion D, Unger T, Lewis DA, Levitt $P$, Mirnics K. Molecular evidence for increased expression of genes related to immune and chaperone function in the prefrontal cortex in schizophrenia. Biol Psychiatry 2007; 62: 711-721.

28. Middleton FA, Mirnics K, Pierri JN, Lewis DA, Levitt P. Gene expression profiling reveals alterations of specific metabolic pathways in schizophrenia. J Neurosci 2002; 22: 27182729.

29. Braak H, Del Tredici K, Sandmann-Kiel D, Rub U, Schultz C. Nerve cells expressing heatshock proteins in Parkinson's disease. Acta Neuropathol 2001; 102: 449-454.

30. Bajramovic JJ, Lassmann H, van Noort JM. Expression of alphaB-crystallin in glia cells during lesional development in multiple sclerosis. J Neuroimmunol 1997; 78: 143-151.

31. Stoevring B, Vang $\mathrm{O}$, Christiansen M. AlphaB-crystallin in cerebrospinal fluid of patients with multiple sclerosis. Clin Chim Acta 2005; 356: 95-101.

32. Shinohara H, Inaguma $\mathrm{Y}$, Goto S, Inagaki T, Kato K. Alpha B crystallin and HSP28 are enhanced in the cerebral cortex of patients with Alzheimer's disease. J Neurol Sci 1993; 119: 203-208.

33. Renkawek K, Voorter CE, Bosman GJ, van Workum FP, de Jong WW. Expression of alpha B-crystallin in Alzheimer's disease. Acta Neuropathol 1994; 87: 155-160.

34. Dabir DV, Trojanowski JQ, Richter-Landsberg C, Lee VM, Forman MS. Expression of the small heat-shock protein alphaB-crystallin in tauopathies with glial pathology. Am J Pathol 2004; 164: 155-166.

35. Lowe J, McDermott H, Pike I, Spendlove I, Landon M, Mayer RJ. alpha B crystallin expression in non-lenticular tissues and selective presence in ubiquitinated inclusion bodies in human disease. J Pathol 1992; 166: 61-68.

36. Ojha J, Karmegam RV, Masilamoni JG, Terry AV, Cashikar AG. Behavioral defects in chaperone-deficient Alzheimer's disease model mice. PLoS One 2011; 6: e16550.

37. Ousman SS, Tomooka BH, van Noort JM, Wawrousek EF, O'Connor KC, Hafler DA et al. Protective and therapeutic role for alphaB-crystallin in autoimmune demyelination. Nature 2007; 448: 474-479.

38. Arac A, Brownell SE, Rothbard JB, Chen C, Ko RM, Pereira MP et al. Systemic augmentation of alphaB-crystallin provides therapeutic benefit twelve hours post-stroke onset via immune modulation. Proc Natl Acad Sci USA 2011; 108: 13287-13292. 
39. Hagemann TL, Boelens WC, Wawrousek EF, Messing A. Suppression of GFAP toxicity by alphaB-crystallin in mouse models of Alexander disease. Hum Mol Genet 2009; 18: 1190-1199.

40. Rao NA, Saraswathy S, Wu GS, Katselis GS, Wawrousek EF, Bhat S. Elevated retinaspecific expression of the small heat shock protein, alphaA-crystallin, is associated with photoreceptor protection in experimental uveitis. Invest Ophthalmol Vis Sci 2008; 49: 1161-1171.

41. Masilamoni JG, Jesudason EP, Baben B, Jebaraj CE, Dhandayuthapani S, Jayakumar R. Molecular chaperone alpha-crystallin prevents detrimental effects of neuroinflammation. Biochim Biophys Acta 2006; 1762: 284-293.

42. Ninkovic J, Pinto L, Petricca S, Lepier A, Sun J, Rieger MA et al. The transcription factor Pax6 regulates survival of dopaminergic olfactory bulb neurons via crystallin alphaA Neuron 2010; 68: 682-694.

43. Liedtke T, Schwamborn JC, Schroer U, Thanos S. Elongation of axons during regeneration involves retinal crystallin beta b2 (crybb2). Mol Cell Proteomics 2007; 6: 895-907.

44. Jobby MK, Sharma Y. Calcium-binding to lens betaB2- and betaA3-crystallins suggests that all beta-crystallins are calcium-binding proteins. FEBS J 2007; 274: 4135-4147.

45. Rajini B, Shridas P, Sundari CS, Muralidhar D, Chandani S, Thomas F et al. Calcium binding properties of gamma-crystallin: calcium ion binds at the Greek key beta gammacrystallin fold. J Biol Chem 2001; 276: 38464-38471.

46. Sharma Y, Rao CM, Narasu ML, Rao SC, Somasundaram T, Gopalakrishna A et al. Calcium ion binding to delta- and to beta-crystallins. The presence of the 'EF-hand' motif in delta-crystallin that aids in calcium ion binding. J Biol Chem 1989; 264: 12794-12799.

47. Wang YH, Wang DW, Wu N, Wang Y, Yin ZQ. Alpha-crystallin promotes rat retinal neurite growth on myelin substrates in vitro. Ophthalmic Res 2010; 45: 164-168.

48. Adhikari AS, Singh BN, Rao KS, Rao Ch M. AlphaB-crystallin, a small heat shock protein, modulates NF-kappaB activity in a phosphorylation-dependent manner and protects muscle myoblasts from TNF-alpha induced cytotoxicity. Biochim Biophys Acta 2011; 1813 1532-1542.

49. Steinman L. A molecular trio in relapse and remission in multiple sclerosis. Nat Rev Immunol 2009; 9: 440-447.

50. Wu N, Wang YH, Zhao HS, Liu DN, Ying X, Yin ZQ et al. Alpha-crystallin downregulates the expression of TNF-alpha and iNOS by activated rat retinal microglia in vitro and in vivo. Ophthalmic Res 2009; 42: 21-28.

51. Fatemi SH, Pearce DA, Brooks Al, Sidwell RW. Prenatal viral infection in mouse causes differential expression of genes in brains of mouse progeny: a potential animal model for schizophrenia and autism. Synapse 2005; 57: 91-99.
52. Asp L, Beraki S, Aronsson F, Rosvall L, Ogren SO, Kristensson K et al. Gene expression changes in brains of mice exposed to a maternal virus infection. NeuroReport 2005; 16: 1111-1115.

53. Lassen N, Bateman JB, Estey T, Kuszak JR, Nees DW, Piatigorsky J et al. Multiple and additive functions of ALDH3A1 and ALDH1A1: cataract phenotype and ocular oxidative damage in Aldh3a1(-/-)/Aldh1a1(-/-) knock-out mice. J Biol Chem 2007; 282: $25-25$.

54. Duester G. Retinoic acid synthesis and signaling during early organogenesis. Cell 2008 134: 921-931.

55. He M, Kratz LE, Michel JJ, Vallejo AN, Ferris L, Kelley RI et al. Mutations in the human SC4MOL gene encoding a methyl sterol oxidase cause psoriasiform dermatitis, microcephaly, and developmental delay. J Clin Invest 2011; 121 976-984.

56. Brown NL, Dagenais SL, Chen CM, Glaser T. Molecular characterization and mapping of $\mathrm{ATOH}$, a human atonal homolog with a predicted role in retinal ganglion cell development. Mamm Genome 2002; 13: 95-101.

57. De Miranda J, Yaddanapudi K, Hornig M, Villar G, Serge R, Lipkin WI. Induction of Toll-like receptor 3-mediated immunity during gestation inhibits cortical neurogenesis and causes behavioral disturbances. MBio 2010; 1: e00176-10.

58. Wolf SA, Melnik A, Kempermann G. Physical exercise increases adult neurogenesis and telomerase activity, and improves behavioral deficits in a mouse model of schizophrenia. Brain Behav Immun 2011; 25: 971-980.

59. Soumiya H, Fukumitsu H, Furukawa S. Prenatal immune challenge compromises development of upper-layer but not deeper-layer neurons of the mouse cerebral cortex. J Neurosci Res 2011; 89: 1342-1350.

60. Deng MY, Lam S, Meyer U, Feldon J, Li Q, Wei R et al. Frontal-subcortical protein expression following prenatal exposure to maternal inflammation. PLOS One 2011; 6: e16638.

Translational Psychiatry is an open-access journal published by Nature Publishing Group. This work is licensed under the Creative Commons Attribution-Noncommercial-No Derivative Works 3.0 Unported License. To view a copy of this license, visit http://creativecommons.org/licenses/by-nc-nd/3.0/

\section{Supplementary Information accompanies the paper on the Translational Psychiatry website (http://www.nature.com/tp)}

